\title{
Alfabetización académica de los estudiantes de Geología y Paleontología de una universidad pública argentina ${ }^{1}$
}

\author{
María Angélica Diez ${ }^{2}$ \\ Instituto de Investigación en Paleobiología y Geología (Universidad Nacional de Río Negro -CONICET), \\ Sede Alto Valle - Valle Medio, General Roca, Río Negro, Argentina \\ David Alberto Londoño-Vásquez 3 \\ Facultad de Ciencias Sociales, Institución Universitaria de Envigado, \\ Envigado, Antioquia, Colombia \\ Norma Cech ${ }^{4}$ \\ Instituto de Investigación en Paleobiología y Geología (Universidad Nacional \\ de Río Negro -CONICET), Sede Alto Valle - Valle Medio, General Roca, Río \\ Negro, Argentina
}

Resumen: Los diferentes niveles de alfabetización académica inicial de los estudiantes universitarios tienen una relación directa con el desempeño en sus primeros años de formación académica. A partir de esta afirmación se investigaron estos niveles en los estudiantes que ingresaron a las Licenciaturas en Geología y en Paleontología en 2018 y 2019 en una universidad argentina. Se diseñó una prueba de entrada y otra de salida, así como una rúbrica de revisión que respondía a los objetivos de la evaluación. Los resultados obtenidos se analizaron cuantitativamente. Las actividades de resumen de un artículo de divulgación científica en las pruebas de entrada señalaron dificultades en los niveles de alfabetización académica. Comparativamente con las de salida se observaron avances en el dominio de la comprensión y producción textual; sin embargo, recién se vuelven significativos cuando los estudiantes transitaron un año de estudios universitarios, acompañados en varias actividades desde el Área de Comunicación Múltiple.

Palabras-clave: Prácticas discursivas; Enseñanza universitaria; Comunicación académica; Alfabetización académica; Ciencias de la Tierra.

\footnotetext{
${ }^{1}$ Este artículo es un resultado parcial del Proyecto de investigación "Innovación pedagógica en Geociencias y construcción de nuevos perfiles docentes en la enseñanza universitaria" (Código PI 40-A-619, Universidad Nacional de Río Negro, Argentina).

2 Doctora en Historia (Universidad Nacional de La Plata), Universidad Nacional de Río Negro, Sede Alto Valle y Valle Medio, General Roca, Argentina. Orcid: https://orcid.org/0000-0002-3574-9391.

E-mail: mdiez@unrn.edu.ar

${ }^{3}$ Doctor en Ciencias Sociales, Niñez y Juventud (Universidad de Manizales-Cinde). Institución Universitaria de Envigado. Orcid: https://orcid.org/0000-0003-1110-7930.

E-mail: dalondono@correo.iue.edu.co

${ }^{4}$ Doctora en Ciencias Biológicas (Universidad Nacional de Córdoba). Universidad Nacional de Río Negro, Sede Alto Valle y Valle Medio, General Roca, Argentina. Orcid: https://orcid.org/0000-0002-6755-5321.

E-mail: ncech@unrn.edu.ar
} 
Título: Alfabetização acadêmica de estudantes de geologia e paleontologia de uma universidade argentina

Resumo: Os diferentes níveis de alfabetização acadêmica inicial dos estudantes universitários têm uma relação direta com o desempenho nos primeiros anos de formação acadêmica. Com base nessa afirmação, esses níveis foram investigados em estudantes que ingressaram no Bacharelado em Geologia e Paleontologia em 2018 e 2019 em uma universidade argentina. Um teste de entrada e um teste de saída foram projetados, bem como uma rubrica de revisão que respondia aos objetivos da avaliação. Os resultados obtidos foram analisados quantitativamente. As atividades resumidas de um artigo de divulgação científica nos vestibulares indicaram dificuldades nos níveis de alfabetização acadêmica. Comparativamente aos resultados, foram observados avanços no domínio da compreensão e produção textual; no entanto, elas só se tornam significativas quando os estudantes passam por um ano de estudos universitários, acompanhados em várias atividades da Área de Comunicação Múltipla.

Palavras-chave: Práticas discursivas; Educação universitária; Comunicação acadêmica; Alfabetização acadêmica; Ciências da terra.

Title: Academic literacy of Geology and Paleontology students in an Argentina public university

Abstract: The different levels of initial academic literacy of university students have a direct relationship with performance in their first years of academic training. Based on this belief, these levels were investigated in students who entered the Bachelor's degrees in Geology and Paleontology in 2018 and 2019 at an Argentine university. An entrance and exit tests were designed, as well as a review rubric that responded to the objectives of the evaluation. The results obtained were analyzed quantitatively. The elaboration of an abstract from of an article of scientific dissemination in the entrance exams indicated difficulties in the levels of academic literacy. Comparatively with the output ones, advances were observed in the domain of textual comprehension and production; however, they only became significant when the students went through a year of university studies, accompanied in several activities from the Multiple Communication Area.

Keywords: Discursive practices; University education; Academic communication; Academic literacy; Earth sciences.

\section{Introducción}

La alfabetización académica resulta una preocupación permanente en las Licenciaturas en Geología y en Paleontología de la Universidad Nacional de Río Negro (UNRN) desde el inicio de estas carreras en el 2010, cuando se observaron las dificultades en lectura, escritura y oratoria de buena parte de los estudiantes. Estas dificultades se ven agravadas por el bajo nivel de educación científica, cuestión fundamental para los aprendizajes en Ciencias de la Tierra ${ }^{5}$. En 2016, a partir de un Proyecto de Innovación Pedagógica (PIP) centrado en la enseñanza de las Geociencias ${ }^{6}$, se dio mayor relevancia a las

\footnotetext{
${ }^{5}$ Las propuestas curriculares del secundario en Argentina no incluyen geología ni paleontología, si bien algunos contenidos pueden estar presentes de manera marginal en diversas materias. Recién en 2018 se implementó en la provincia de Río Negro una reforma que incluyó dos espacios curriculares específicos, como Ciencias de la Tierra 1 y 2, en la orientación Bachiller en Ciencias Naturales (ARGENTINA, GOBIERNO DE RÍO NEGRO, 2017).

${ }^{6}$ A fines de 2015 la Licenciatura en Geología de la Universidad Nacional de Río Negro, por pertenecer al grupo
} 
cuestiones de lectura, escritura y oratoria para cualquiera de las disciplinas que integran estos programas (DIEZ; CASADÍO, 2018). Estas carreras incluyen en primer año (primer cuatrimestre) la asignatura Introducción a la Lectura y Escritura Académica (en adelante ILEA); sin embargo, los docentes consideran que no resulta suficiente para lograr un mejoramiento cualitativamente significativo de estas prácticas. El equipo de cátedra resolvió desde el 2016 colaborar en otros momentos de los trayectos formativos de los y las estudiantes, especialmente durante el segundo cuatrimestre de primer año, a fin de consolidar y profundizar saberes de comprensión y producción textual y de oratoria. Este trabajo de acompañamiento lo realizan tanto a nivel docente como estudiantil.

En el 2018 se acreditaron dos proyectos de investigación destinados a profundizar en los procesos de innovación educativa y, a partir de ellos, no solo se planteó la preocupación por la alfabetización académica, sino que se comenzó a hacer hincapié en los niveles de comprensión y producción textual de los estudiantes que ingresan a estas carreras de Ciencias de la Tierra. Esta propuesta implica dar mayor relevancia al contexto del proceso de lectura y de producción escritural, así como tener presente la competencia de criticidad que estas acciones debieran contener no solo para lo disciplinar, sino para la formación de un pensamiento crítico como ciudadanos (LONDOÑO; BERMÚDEZ, 2018).

Los resultados que aquí se presentan responden a un primer análisis cuantitativo del problema de la comprensión lectora y la producción de textos escritos que manifiestan los estudiantes de Geología y de Paleontología de la UNRN -cohortes 2018 y 2019- durante los primeros 12 meses formativos, realizados a partir de pruebas de entrada y de salida. Cada actividad de las pruebas, una de resumen y otra de lectura crítica, contenía dos partes de las cuales una remitía a conocimientos de tipo teóricos, y la otra estaba centrada en el desarrollo práctico de la lectura y escritura; sin embargo, muy pocos estudiantes de cada cohorte pudieron realizar las cuatro secciones de la prueba.

En general, los estudiantes abordaron solo la actividad del resumen realizando una o ambas secciones, mientras que, menos estudiantes resolvieron también las dos cuestiones referidas a la lectura crítica, con la salvedad de la prueba de salida de la cohorte 2018 . Esto pudo deberse a que la lectura crítica les presentó mayores dificultades, o bien a que siguieron el orden de la guía en donde lo referido al resumen estaba en primero lugar y que, para el desempeño de la mayoría de los y las estudiantes, las dificultades para la lectura no les permitió que el tiempo otorgado les fuera suficiente. Así mismo, los problemas para la

de carreras declaradas de interés público, fue evaluada por la Comisión Nacional de Evaluación y Acreditación Universitaria (CONEAU), organismo descentralizado que funciona en la jurisdicción del Ministerio de Educación de la Nación. Como resultado la carrera fue acreditada por 6 años, concluido este plazo deberá ser nuevamente evaluada (Resolución 284/16 - CONEAU). En el proyecto institucional presentado a la CONEAU la propuesta pedagógica avanzaba con mayor innovación sobre los pilares ya establecidos en el inicio la carrera en 2010. Una cuestión central fue que, las asignaturas se integraran en torno a problemas o proyectos de estudio diseñados por los docentes en forma mancomunada. A partir de esto, la materia Introducción a la Lectura y Escritura Académica (ILEA) trabajó en el planteo de un Área de Comunicación Múltiples, asumiendo objetivos más amplios en los procesos de enseñanza y aprendizaje de los demás espacios curriculares. 
lectura crítica quedaron manifestadas en quienes sí intentaron resolver la actividad. Para este artículo se analiza centralmente el resumen resultado de la lectura de un artículo de divulgación.

\section{Alfabetización académica en el proceso de formación universitaria}

Un número significativo de comunidades en Latinoamérica ha venido ganando espacios frente al acceso a la educación universitaria (HERNÁNDEZ et al., 2015; FERNÁNDEZ; PÉREZ, 2016); puesto que, personas de diferentes niveles sociales, económicos y culturales han podido acceder a una formación superior desde fines de la década de $1990 .{ }^{7}$ Lo anterior se ha traducido en la participación de personas con una mayor diversidad de intereses y de diferenciación de conocimientos previos y capacidades que buscan formarse en algún área del conocimiento (MESSINA; SILVA, 2017; FAUSTO et al., 2018). Sin embargo, dependiendo del programa académico y de la universidad a la cual desean acceder, las posibilidades de ingreso pueden verse reducidas. Esto significa que hay carreras con un número muy elevado de candidatos y con uno inferior de cupos (YUNI et al., 2014; DI CAUDO, 2016). Además, en las universidades públicas latinoamericanas, en general se plantea un proceso de admisión relacionado con pruebas de ingreso o calidades académicas previas evidenciadas en el historial del candidato (SORIA; ZÚÑIGA, 2014; TAPASCO et al., 2016).

En Argentina, uno de los países con mayor porcentaje de estudiantes universitarios desde la década de 1990 en Latinoamérica (AROCENA; SUTZ, 2001) y en general con ingreso irrestricto a las universidades ${ }^{8}$, ha venido planteando y desarrollando investigaciones educativas acerca de cuestiones y problemas referidos a la formación universitaria. Los más recurrentes son: igualdad en el acceso a la educación (MARTÍNEZ, 2011), ingreso a la universidad pública (CATINO; JUARROS, 2011), ingreso condicionado (GLUZ; ROSICA, 2011), permanencia en la universidad (WEBER, 2011) y deserción (FALCONE; STRAMAZZI, 2011). Estos temas convergen en el papel de la educación para la formación del estudiante y futuro profesional en interacción con los requerimientos y las problemáticas de la sociedad, reconociendo las potencialidades que puede tener no solo en su proyecto de vida personal, sino también en la afectación de su entorno y en las posibilidades sociales, culturales y

\footnotetext{
${ }^{7}$ Arocena y Sutz (2001) proponen que la tasa de escolarización superior en América Latina creció de 1.9\% en 1950 a $20.7 \%$ en 1994, siendo Argentina (41.8\%), Costa Rica (33.1\%), Perú (31.2) y Chile (30.3) los países con mayor porcentaje de estudiantes graduados de secundaria matriculados en un programa de educación de tercer nivel para 1996. Según el Banco Mundial, en Latinoamérica, en el 2013, la tasa de escolarización fue de 43\% (Smink, 2017). Es importante mencionar que, para ese mismo año, la tasa de deserción en esta región fue del $50 \%$ y que las comunidades con más bajos recursos representaron el $24 \%$ de los estudiantes matriculados.

8 En general en las universidades públicas argentinas el ingreso es irrestricto y, en los casos en que hay cursos de ingreso, aun cuando incluyan evaluaciones, tienen por finalidad dar una base de conocimientos y nivelar los saberes, es decir que no dejan fuera del sistema a los y las estudiantes que no tienen los mínimos saberes y competencias para el estudio universitario. Así mismo pueden ingresar sin tener todas las materias aprobadas en el secundario, variando el número permitido entre las distintas universidades.
} 
económicas de la sociedad.

Otro aspecto que aparece entre las preocupaciones de las autoridades educativas y los investigadores argentinos frente a la educación universitaria son los niveles de lectura y escritura en los procesos de formación de los estudiantes (CARLINO, 2011). No solo como una aspiración de lo deseable, sino como un proceso de alfabetización académica que debe ser constantemente trabajado desde las diferentes disciplinas a fin de permitir a los y las estudiantes acceder y construir los procesos apropiados para los aprendizajes disciplinarios. Este trabajo está relacionado con los textos que leen, acompañados de preguntas que los guíen en la comprensión lectora y la construcción de la escritura. Así mismo, se enriquece cuando se facilita la creación de espacios de reflexión, retroalimentación y construcción discursiva disciplinaria. Entre estas opciones también surgen propuestas de orientación tanto para el acceso a una comprensión lectora disciplinar, como para la producción de textos escritos, como son las del acompañamiento de estudiantes con mayor experiencia escritural y disciplinaria, o bien por parte de tutores o docentes del área (CARLINO, 2005).

En relación a lo disciplinario, se encuentran diversas propuestas y experiencias a partir de la conformación de equipos interdisciplinarios integrados por docentes de la lengua y de las diferentes disciplinas de los programas académicos, con el objetivo de fortalecer la alfabetización académica a través de una adecuada selección de los textos a trabajar en los cursos y, además, hacerlo de forma mancomunada. Así mismo, comparten el diseño de las actividades de comprensión lectora y producción textual que se van a solicitar a los estudiantes (NATALE, 2012), preparan documentos de apoyo que permiten a los estudiantes trabajar diferentes producciones textuales académicas (o géneros discursivos) como la reseña académica (NAVARRO; ABRAMOVICH, 2012), el ensayo académico (ZUNINO; MURACA, 2012), el estado de la cuestión (BENGOCHEA; LEVÍN, 2012), el proyecto de intervención (STAGNARO; DA REPRESENTACAO, 2012), entre otros.

En la UNRN los problemas de lectura y escritura, como se mencionó anteriormente, han sido una preocupación constante. Desde los inicios institucionales en 2009 uno de los cursos de ingreso estaba destinado a nivelar a los estudiantes en sus saberes y capacidades para la lectura y escritura, y para iniciarlos en las formas académicas de hacerlo. A la vez algunas carreras establecieron en sus planes de estudio una asignatura con este mismo fin. Con posterioridad, se fueron cambiando los formatos de la oferta de cursos de ingresos y asumieron esta decisión las propias carreras, desapareciendo estos curso en algunas y manteniéndolos en otras, al igual que una materia específica de comprensión y producción textual en primer año. En una década de existencia institucional, los resultados de las iniciativas institucionales no resultan plenamente satisfactorios, lo que ha llevado a ir variando las propuestas en busca de alternativas que realmente mejoren la situación de aprendizaje de los estudiantes. Últimamente en muchas carreras se resolvió incluir o modificar la asignatura de lectura y escritura académica, dándole un enfoque orientado a la disciplina profesional que corresponda. 
En las Licenciaturas en Geología y en Paleontología de la UNRN se realizan experiencias de innovación pedagógica que apuntan a fortalecer y enriquecer los procesos de aprendizaje y de formación académica en vistas de los perfiles profesionales deseables, su inserción laboral y su responsabilidad con el entorno y la sociedad. Así también se contempla el perfil general del estudiantado y, al igual que el que predomina en toda la Universidad Nacional de Río Negro:

\begin{abstract}
Los datos sociodemográficos de los alumnos ingresantes evidencian que mayoritariamente provienen de la provincia de Rio Negro, son primera generación de universitarios, trabajan, el porcentaje que se corresponde con la edad teórica de ingreso a los estudios universitarios es bajo y la tasa de feminización es alta en todas las sedes. En el año 2015 el 76\% de los estudiantes es de Río Negro, concurriendo el $69 \%$ a la Sede Alto Valle y Valle Medio (...). En la serie 2009, 2012 y 2015 el porcentaje para la UNRN de ingresantes primera generación de universitarios se mantiene relativamente estable ascendiendo al 71,8\%, 69,9\% y 66,9\% (...)." (CONEAU 2018).
\end{abstract}

Esta impronta de nuevos inscriptos que son primera generación de estudiantes universitarios de sus familias, se mantiene en porcentaje elevado, siendo del $60 \%$ en 2018 (UNIVERSIDAD NACIONAL DE RÍO NEGRO 2018).

En estas carreras y desde el inicio de estas carreras en 2010 se fomentó un trabajo transversal entre diversas asignaturas, con la finalidad de construir un proceso integral entre los conocimientos y desarrollo de capacidades del buen uso de la lengua, y los aspectos comunicacionales propios de las disciplinas centrales y auxiliares de las Ciencias de la Tierra. En este sentido, se reconoce que:

el desarrollo de habilidades para escribir textos académicos (...) exige un entrenamiento de alta complejidad. (...). Estas actividades, por otra parte, se desarrollan en situaciones comunicativas nuevas, intervienen lectores $\mathrm{y} / \mathrm{o}$ evaluadores expertos, diferentes contextos institucionales y por lo tanto, los escritos que reflejan estas tareas científicas deben corresponder a lo que la comunidad de discurso de la disciplina en la que se escribe ha establecido. Estas situaciones comunicativas convencionalizadas se corresponden con géneros o clases de discursos con características específicas y exigen nuevas competencias comunicativas. (CUBO DE SEVERINO Y CASTRO DE CASTILLO, 2005, p. 15).

En el 2016 la asignatura ILEA expandió en la práctica sus límites curriculares con la idea de conformar un área que asumió la denominación de Área de Comunicación Múltiple y que pueda ser establecida como tal en el diseño de un nuevo Plan de Estudio para la Licenciatura en Geología. De esta manera, se vienen diseñando actividades de lectura, escritura, expresión oral y diseño de producciones visuales y audiovisuales entre docentes del área de comunicación y los de materias disciplinares en torno a: lectura de artículos de divulgación científica; reconocimiento de la estructura y del estilo de escritura en los artículos científicos; elaboración de circulares, poster académico y su resumen para un simulacro de evento científico; presentaciones orales con pautas de oratoria y diseño de soportes visuales o audiovisuales; escritura, dibujo y representación gráfica en libretas de 
campo; argumentación matemática escrita y oral; recursos analógicos para la expresión matemática; entre otras. Todas ellas contienen saberes y capacidades que hacen a las prácticas centrales en la formación y el ejercicio profesional en Geología y Paleontología (DIEZ et al. 2017; GONZÁLEZ et al., 2017; CÁBANA et al. 2017; DIEZ et al. 2018).

Este transcurrir desde el inicio de las carreras de Geología y Paleontología, aún con los cambios implementados a partir de 2016, sigue dando cuenta de dificultades. Por una parte, desde el dominio de saberes de parte de los y las estudiantes con que ingresan a la universidad; por la otra, la falta de un claro reconocimiento por parte de los docentes de las claves que particularizan la lectura y escritura desde sus disciplinas, así como la ausencia de formación docente en este sentido. Ambas cuestiones, llevaron a plantear la investigación de cuestiones de alfabetización académica en el campo de la enseñanza y los aprendizajes en Geociencias.

\section{Métodos y técnicas}

Este trabajo se inserta en una investigación más amplia que aborda el problema de la alfabetización académica en el campo de la enseñanza y los aprendizajes en Geociencias con un método mixto que combina el enfoque cualitativo (hermenéutico-comprensivo) con el cuantitativo que es el que se aplicó en el estudio realizado para este artículo. El diseño se adecuó a una investigación diagnóstica de tipo exploratoria, apoyada en herramientas estadísticas con un objetivo primordialmente descriptivo a partir de los resultados en cifras y porcentajes que fueron representados en tablas y gráficos. Esto permitió realizar un análisis descriptivo global y, luego, uno específico de los rasgos caracterizados por los resultados más destacados, generando una comparación en dos direcciones, una diacrónica para una misma cohorte de estudiantes en dos momentos (prueba de entrada y de salida), y otra sincrónica entre ambas cohortes (2018 y 2019).

El problema de investigación se centró en identificar los niveles de comprensión textual que tienen los participantes y las cualidades textuales de las producciones escritas de los mismos. Para ello, se diseñaron dos pruebas: una de entrada y otra de salida, basadas en los requerimientos para la comprensión y producción textual para estudiantes universitarios propuestos por HENAO et al. (2011). De los 14 requerimientos propuestos, se seleccionaron los siguientes:

1. Diferenciar los tipos de significados y los diferentes tipos de textos.

2. Resumir usando las macrorreglas.

3. Leer críticamente.

4. Escribir con cohesión, coherencia, adecuación y corrección.

5. Producir textos adaptables, eficaces, eficientes y con información nueva.

6. Producir textos con la estructura adecuada. 
7. Citar y referenciar de acuerdo a las exigencias de las publicaciones.

8. Argumentar desde una posición pragmadialéctica, evitando falacias argumentativas.

Dichas pruebas se componían de cuatro instrucciones a partir de dos textos que remiten al contexto regional, productivo y laboral de la Patagonia argentina y chilena, y pertenecen al área de conocimientos de las Ciencias de la Tierra. Los mismos fueron seleccionados a partir de una serie de materiales proporcionados por docentes de estas ciencias. Las instrucciones fueron:

1. Resumir un artículo de divulgación. Los textos elegidos para cada prueba son narrativos-descriptivos con significados literales, implícitos y complementarios (ARGAÉZ; ESPINOZA 2004; GALLEGOS 2015).

2. Mencionar cómo se realizó el resumen.

3. Realizar una lectura crítica de una columna de opinión. Como tales fueron textos argumentativos-descriptivos, con una amplia carga ideológica y rico en significados implícitos y complementarios (BERDICHEVSKY 2017; MONREAL 2018).

4. Mencionar qué se entiende por lectura crítica.

Las pruebas contaban con una rúbrica para su análisis. Allí se empleó la propuesta por LONDOÑO (2016), la cual contiene 20 variables que están relacionadas con la calidad textual y discursiva esperadas en la elaboración de un resumen y un texto argumentativo como producto de una lectura crítica. La rúbrica fue validada por integrantes del proyecto, quienes por otra parte integran la cátedra de ILEA.

El trabajo cuantitativo observacional descriptivo se realizó en base a dos cohortes de ingreso (2018 y 2019) ${ }^{9}$, permitiendo el tratamiento de los datos obtenidos y su representación gráfica. Se entiende por cohorte un grupo con ciertas características similares consideradas así desde los criterios de la investigación. En este caso: estudios secundarios completos o por concluir, intereses similares por la elección de carreras de Ciencias de la Tierra, y ser ingresantes.

Cada cohorte abarcó el número de estudiantes inscriptos en las carreras de Geología y de Paleontología y, a su vez, matriculados en la asignatura de Introducción a la Lectura y Escritura Académica (ILEA) que es la que asume la realización de las pruebas como diagnósticos de inicio de la cursada. Para la cohorte 2018, según los datos institucionales ofrecidos por el Departamento de alumnos (Sede Alto Valle - Valle Medio), los inscriptos en la carrera fueron 76 estudiantes (63 de Geología y 13 de Paleontología), de los cuales 31 (41\%) siguieron activos al momento la prueba de salida realiza al inicio de segundo año de la carrera. ${ }^{10}$ Mientras que, en la cohorte 2019 se inscribieron 66 estudiantes (52 de Geología y

\footnotetext{
${ }^{9}$ Dado los posibles problemas en las interpretaciones que podría acarreas la observación por cohorte, se optó por analizar dos cohortes con continuidad temporal, en vez de tomar una aisladamente; por otra parte, porque las cohortes no existen en tal aislamiento.

${ }^{10}$ En Argentina ingresan a la universidad con 13 años de escolaridad obligatoria distribuidos en Nivel Inicial, Primaria y Secundaria; es decir que, el general acceden a la universidad entre los 17 y 18 años.
} 
14 de Paleontología), de los cuales 44 (67\%) continuaron activos al inicio del segundo cuatrimestre cuando se realizó la prueba de salida.

El número de los y las estudiantes quienes realizaron las pruebas resultó inferior a los inscriptos en ILEA, unos pocos por inasistencia, y la mayoría debido a que habían abandonado las carreras antes de iniciar los cursos (tabla 1). Para ambas cohortes se realizaron las pruebas de salida, para las cuales el número de estudiantes evaluados disminuyó debido a los ausentes, la deserción y a que, aún sin abandonar, algunos quedaron rezagados en sus estudios ${ }^{11}$.

Tabla 1 - Estudiantes que realizaron las pruebas de entrada y de salida.

Cohortes 2018 y 2019.

\begin{tabular}{|c|c|c|c|c|c|c|c|c|}
\hline \multirow[b]{2}{*}{ Cohorte } & \multicolumn{4}{|c|}{ Prueba de entrada } & \multicolumn{4}{|c|}{ Prueba de salida } \\
\hline & $\begin{array}{l}\text { Técnica } \\
\text { resumen }\end{array}$ & $\begin{array}{c}\text { Resumen } \\
\text { de texto }\end{array}$ & $\begin{array}{c}\text { Concepto } \\
\text { L.C. }\end{array}$ & $\begin{array}{c}\text { L.C. del } \\
\text { texto }\end{array}$ & $\begin{array}{l}\text { Técnica } \\
\text { resumen }\end{array}$ & $\begin{array}{l}\text { Resumen } \\
\text { del texto }\end{array}$ & $\begin{array}{c}\text { Concepto } \\
\text { L.C. }\end{array}$ & $\begin{array}{c}\text { L.C. del } \\
\text { texto }\end{array}$ \\
\hline 2018 & 52 & 47 & 19 & 11 & 8 & 8 & 23 & 16 \\
\hline 2019 & 55 & 55 & 18 & 7 & 20 & 20 & 11 & $11^{*}$ \\
\hline
\end{tabular}

Con respecto la revisión de las pruebas a través de las rúbricas, se recurrió a una correctora externa a la cátedra de ILEA y al proyecto de investigación ${ }^{12}$. Los datos resultantes se cargaron en una planilla excel diseñada con este fin, a partir del cual se realizaron las tablas y gráficos. Asimismo, la docente redactó un informe preliminar por tipo de pruebas entrada y salida- y por cohortes -2018 y 2019- que no solo hacía referencias a dichos resultados, sino que planteaba algunas interpretaciones bajo una visión global de cada prueba como correctora externa, no solo de los resultados, sino también sus comentarios acerca del diseño de las pruebas, los textos seleccionados y el tiempo destinado a que los estudiantes realicen las actividades. El mismo se consideró documentación para la investigación, y funcionó como conjeturas parciales surgidas durante el proceso investigativo fortaleciendo la investigación, en particular su proyección futura.

\section{Resultados}

\section{Desempeño en resumen: comparación entre pruebas de entrada y de salida de una misma cohorte}

\footnotetext{
${ }^{11}$ En Geología y de Paleontología de la Universidad Nacional de Río Negro, para la cohorte 2018 los datos institucionales dan cuenta de que en primer año de estas carreras consideradas en conjunto, el $56 \%$ de los nuevos ingresantes no registra aprobación de ninguna materia de las 11, un $18 \%$ aprobó seis o más asignaturas, y un $10 \%$ cinco; el resto se mantiene por debajo de este último número de materias aprobadas.

12 Se convocó para esta colaboración a una profesora con experiencia en realizar este tipo de trabajos por su participación en el proyecto de investigación "Escrituras de (la) emergencia" (2014, Universidad Nacional del Comahue, Neuquén, Argentina), cumpliendo funciones de correctora de protocolos producidos por estudiantes universitarios, función que también cumplió en diversas cátedras.
} 


\section{Cohorte 2018: Resultados globales}

En el desempeño de los y las estudiantes de la cohorte 2018, considerando los resultados globales de las pruebas de entrada y de salida, se observó una mejoría significativa (tablas 2 y 3). Cabe destacar dos cuestiones, por un lado aumentan los resultados esperados, siendo que en 10 de 11 aspectos evaluados el porcentaje supera el $62 \%$, de los cuales dos ascienden al $75 \%$ (coherencia y cohesión) y dos al $88 \%$ (macrorregla suprimir y comprensión de significados). Por el otro, desaparecen los resultados insuficientes, salvo en una categoría (distancia del texto).

Tabla 2 - Técnica de resumen y Resumen de texto. Cohorte 2018. Prueba de entrada.

\begin{tabular}{|c|c|c|c|c|c|c|c|}
\hline & & \multicolumn{3}{|c|}{ Número de individuos } & \multicolumn{3}{|c|}{ Porcentajes } \\
\hline & & \multicolumn{6}{|c|}{ Nivel de desempeño } \\
\hline & & Insuficiente & Aceptable & Esperado & Insuficiente & Aceptable & Esperado \\
\hline Técnica de res & sumen & 28 & 22 & 2 & $54 \%$ & $42 \%$ & $4 \%$ \\
\hline \multirow{4}{*}{ Macrorreglas } & Seleccionar & 4 & 34 & 9 & $9 \%$ & $72 \%$ & $19 \%$ \\
\hline & Suprimir & 1 & 37 & 9 & $2 \%$ & $79 \%$ & $19 \%$ \\
\hline & Generalizar & 7 & 27 & 13 & $15 \%$ & $57 \%$ & $28 \%$ \\
\hline & Integrar & 10 & 27 & 10 & $21 \%$ & $57 \%$ & $21 \%$ \\
\hline \multicolumn{2}{|c|}{ Macrorreglas resultado } & 1 & 33 & 13 & $2 \%$ & $70 \%$ & $28 \%$ \\
\hline \multirow{3}{*}{$\begin{array}{l}\text { Calidad } \\
\text { discursiva }\end{array}$} & Coherencia & 2 & 29 & 16 & $4 \%$ & $62 \%$ & $34 \%$ \\
\hline & Cohesión & 3 & 35 & 9 & $6 \%$ & $74 \%$ & $19 \%$ \\
\hline & $\begin{array}{l}\text { Comprensión } \\
\text { de significados }\end{array}$ & 0 & 22 & 25 & $0 \%$ & $47 \%$ & $53 \%$ \\
\hline \multicolumn{2}{|c|}{ Distancia de texto } & 34 & 5 & 8 & $72 \%$ & $11 \%$ & $17 \%$ \\
\hline \multicolumn{2}{|c|}{ Marcadores de discurso } & 15 & 26 & 6 & $32 \%$ & $55 \%$ & $13 \%$ \\
\hline
\end{tabular}

Tabla 3 - Técnica de resumen y Resumen de texto. Cohorte 2018. Prueba de salida.

\begin{tabular}{|c|c|c|c|c|c|c|c|}
\hline & & \multicolumn{3}{|c|}{ Número de individuos } & \multicolumn{3}{|c|}{ Porcentajes } \\
\hline & & \multicolumn{6}{|c|}{ Nivel de desempeño } \\
\hline & & Insuficiente & Aceptable & Esperado & Insuficiente & Aceptable & Esperado \\
\hline Técnica de res & sumen & 0 & 5 & 3 & $0 \%$ & $62 \%$ & $38 \%$ \\
\hline \multirow{4}{*}{ Macrorreglas } & Seleccionar & 0 & 3 & 5 & $0 \%$ & $38 \%$ & $62 \%$ \\
\hline & Suprimir & 0 & 1 & 7 & $0 \%$ & $12 \%$ & $88 \%$ \\
\hline & Generalizar & 0 & 3 & 5 & $0 \%$ & $38 \%$ & $62 \%$ \\
\hline & Integrar & 0 & 3 & 5 & $0 \%$ & $38 \%$ & $62 \%$ \\
\hline Macrorreglas & resultado & 0 & 3 & 5 & $0 \%$ & $38 \%$ & $62 \%$ \\
\hline \multirow{3}{*}{$\begin{array}{l}\text { Calidad } \\
\text { discursiva }\end{array}$} & Coherencia & 0 & 2 & 6 & $0 \%$ & $25 \%$ & $75 \%$ \\
\hline & Cohesión & 0 & 2 & 6 & $0 \%$ & $25 \%$ & $75 \%$ \\
\hline & $\begin{array}{l}\text { Comprensión } \\
\text { de significados }\end{array}$ & 0 & 1 & 7 & $0 \%$ & $12 \%$ & $88 \%$ \\
\hline \multicolumn{2}{|c|}{ Distancia de texto } & 1 & 5 & 2 & $12 \%$ & $63 \%$ & $25 \%$ \\
\hline \multicolumn{2}{|c|}{ Marcadores de discurso } & 0 & 3 & 5 & $0 \%$ & $38 \%$ & $62 \%$ \\
\hline
\end{tabular}




\section{Cohorte 2018: Resultados particulares}

Al tener que definir las técnicas de resumen utilizadas en las pruebas de entrada más de la mitad de los estudiantes no superaron el nivel de insuficiente (54\%), mientras que para la salida el rendimiento mejoró logrando un 64\% obtener resultados aceptables (gráficos 1 y 2).

Gráfico 1 - Técnicas de resumen. Cohorte 2018 - Prueba de entrada

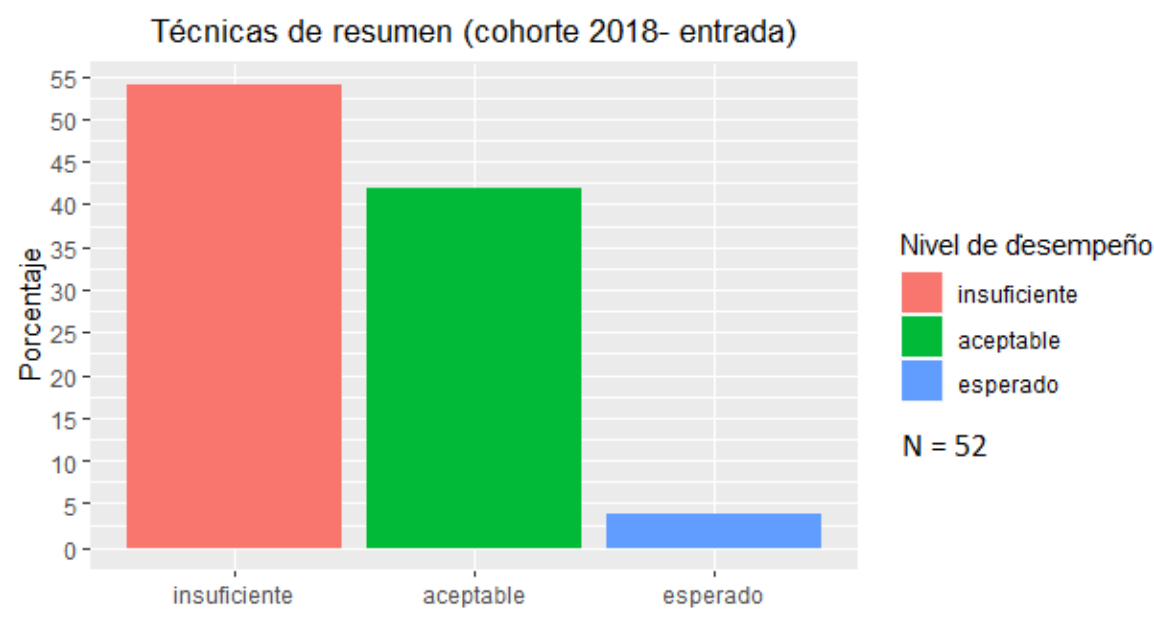

Gráfico 2 - Técnicas de resumen. Cohorte 2018 - Prueba de salida

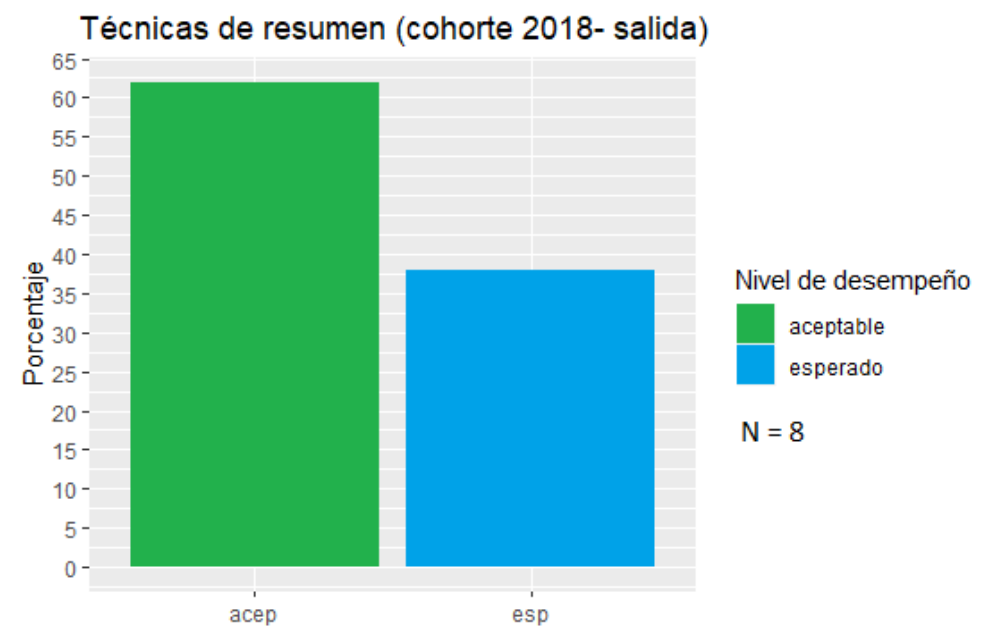

En la realización de los resúmenes pueden destacarse diversas cuestiones (gráficos 3 y 4). El mayor nivel de mejoría lo alcanzan en la calidad discursiva, pues tanto en coherencia, cohesión y comprensión muestran un corrimiento al nivel de esperados en la prueba de salida, ascendiendo al $75 \%$. Así mismo, en el uso de marcadores discursivos con $62 \%$ de resultados esperados. En tanto que, en el uso de las macrorreglas se observan cambios favorables que se reflejan en un mayor nivel de esperado (62\%), mientras que en la prueba 
de entrada el predominio era de resultados aceptables (70\%). Así mismo, desaparecen los resultados insuficientes, salvo en distancia del texto, aunque se destaca un cambio es significativo ya que el índice baja de $72 \%$ a $12 \%$, a la vez que en esperados sube a $63 \%$.

Gráfico 3 - Resumen de texto - Cohorte 2018 - Prueba de entrada

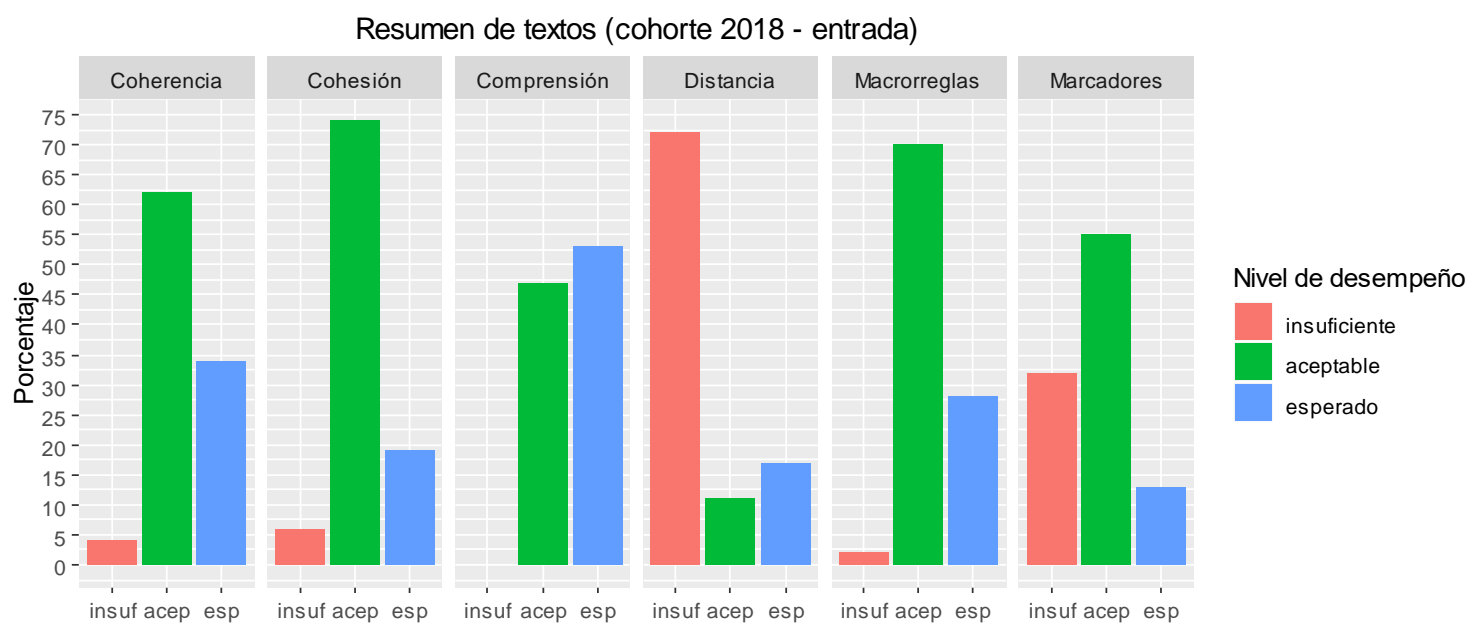

Gráfico 4 - Resumen de texto - Cohorte 2018 - Prueba de salida

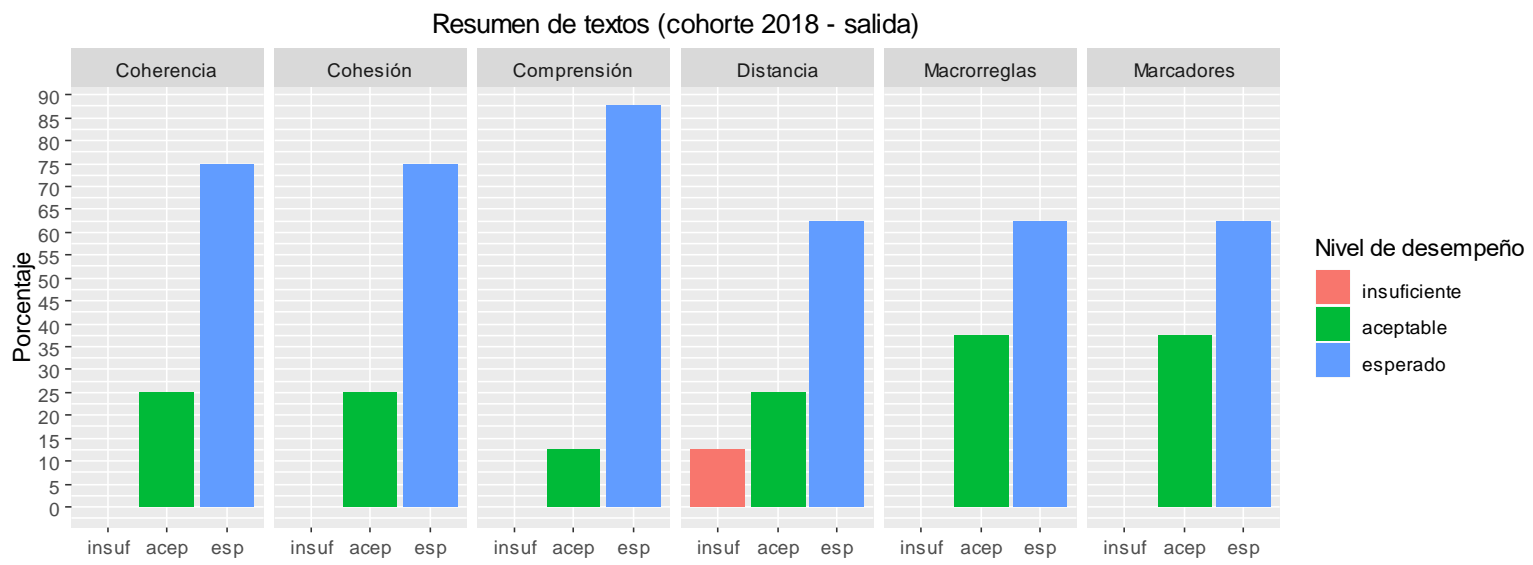

Cohorte 2019: Resultados globales

En el desempeño de los y las estudiantes de la cohorte 2019 considerando los resultados globales se observa una mejoría, pero no se manifiesta de manera homogénea, lo que dificulta su apreciación y síntesis (tablas 4 y 5). Los resultados del nivel esperado subieron en 9 de las 11 categorías evaluadas y los aceptables en 8, mientras que en 6 bajó el nivel de resultados insuficientes. Los cambios favorables más significativos se observaron en distancia del texto al bajar el nivel de insuficientes, diferencia que se repartió en favor de los 
resultados aceptables y los esperados, y en la categoría comprensión en calidad discursiva, en la que no aparece representado el nivel de insuficiente.

Tabla 4 - Técnica de resumen y Resumen de texto. Cohorte 2019. Prueba de entrada.

\begin{tabular}{|c|c|c|c|c|c|c|c|}
\hline & & \multicolumn{3}{|c|}{ Número de individuos } & \multicolumn{3}{|c|}{ Porcentajes } \\
\hline & & \multicolumn{6}{|c|}{ Nivel de desempeño } \\
\hline & & Insuficiente & Aceptable & Esperado & Insuficiente & Aceptable & Esperado \\
\hline Técnica de res & sumen & 17 & 32 & 6 & $31 \%$ & $58 \%$ & $11 \%$ \\
\hline \multirow{4}{*}{ Macrorreglas } & Seleccionar & 27 & 20 & 8 & $49 \%$ & $36 \%$ & $15 \%$ \\
\hline & Suprimir & 15 & 32 & 8 & $27 \%$ & $58 \%$ & $15 \%$ \\
\hline & Generalizar & 10 & 38 & 7 & $18 \%$ & $69 \%$ & $13 \%$ \\
\hline & Integrar & 20 & 30 & 5 & $36 \%$ & $55 \%$ & $9 \%$ \\
\hline \multicolumn{2}{|c|}{ Macrorreglas resultado } & 9 & 39 & 7 & $16 \%$ & $71 \%$ & $13 \%$ \\
\hline \multirow{3}{*}{$\begin{array}{l}\text { Calidad } \\
\text { discursiva }\end{array}$} & Coherencia & 9 & 40 & 6 & $16 \%$ & $73 \%$ & $11 \%$ \\
\hline & Cohesión & 13 & 37 & 5 & $24 \%$ & $67 \%$ & $9 \%$ \\
\hline & $\begin{array}{r}\text { Comprensión } \\
\text { de significados }\end{array}$ & 7 & 42 & 6 & $13 \%$ & $76 \%$ & $11 \%$ \\
\hline \multicolumn{2}{|c|}{ Distancia de texto } & 44 & 7 & 4 & $80 \%$ & $13 \%$ & $7 \%$ \\
\hline \multicolumn{2}{|c|}{ Marcadores de discurso } & 21 & 29 & 5 & $38 \%$ & $53 \%$ & $9 \%$ \\
\hline
\end{tabular}

Tabla 5 - Técnica de resumen y Resumen de texto. Cohorte 2019. Prueba de salida

\begin{tabular}{|c|c|c|c|c|c|c|c|}
\hline & \multicolumn{3}{|c|}{ Número de individuos } & \multicolumn{3}{|c|}{ Porcentajes } \\
\hline & & \multicolumn{6}{|c|}{ Nivel de desempeño } \\
\hline & & Insuficiente & Aceptable & Esperado & Insuficiente & Aceptable & Esperado \\
\hline \multicolumn{2}{|c|}{ Técnica de resumen } & 9 & 11 & 0 & $45 \%$ & $55 \%$ & $0 \%$ \\
\hline \multirow{4}{*}{ Macrorreglas } & Seleccionar & 2 & 11 & 7 & $10 \%$ & $55 \%$ & $35 \%$ \\
\hline & Suprimir & 1 & 13 & 6 & $5 \%$ & $65 \%$ & $30 \%$ \\
\hline & Generalizar & 4 & 13 & 3 & $20 \%$ & $65 \%$ & $15 \%$ \\
\hline & Integrar & 6 & 13 & 1 & $30 \%$ & $65 \%$ & $5 \%$ \\
\hline \multicolumn{2}{|c|}{ Macrorreglas resultado } & 1 & 15 & 4 & $5 \%$ & $75 \%$ & $20 \%$ \\
\hline \multirow{3}{*}{$\begin{array}{l}\text { Calidad } \\
\text { discursiva }\end{array}$} & Coherencia & 4 & 11 & 5 & $20 \%$ & $55 \%$ & $25 \%$ \\
\hline & Cohesión & 7 & 10 & 3 & $35 \%$ & $50 \%$ & $15 \%$ \\
\hline & $\begin{array}{l}\text { Comprensión } \\
\text { de significados }\end{array}$ & 0 & 14 & 6 & $0 \%$ & $70 \%$ & $30 \%$ \\
\hline \multicolumn{2}{|c|}{ Distancia de texto } & 5 & 8 & 7 & $25 \%$ & $40 \%$ & $35 \%$ \\
\hline \multicolumn{2}{|c|}{ Marcadores de discurso } & 8 & 10 & 2 & $40 \%$ & $50 \%$ & $10 \%$ \\
\hline
\end{tabular}

Cohorte 2019: Resultados particulares

Al tener que definir las técnicas de resumen utilizadas en las pruebas de entrada más de la mitad de los y las estudiantes lograron el nivel de aceptable (58\%), observándose un porcentaje similar en las pruebas de salida (gráficos 7 y 8). Sin embargo, en estas últimas no aparecen resultados esperados que en la prueba de entrada ascendían al $11 \%$, y hay un marcado porcentaje de insuficientes (45\%). 
Gráfico 7 - Técnicas de resumen. Cohorte 2019 - Prueba de entrada

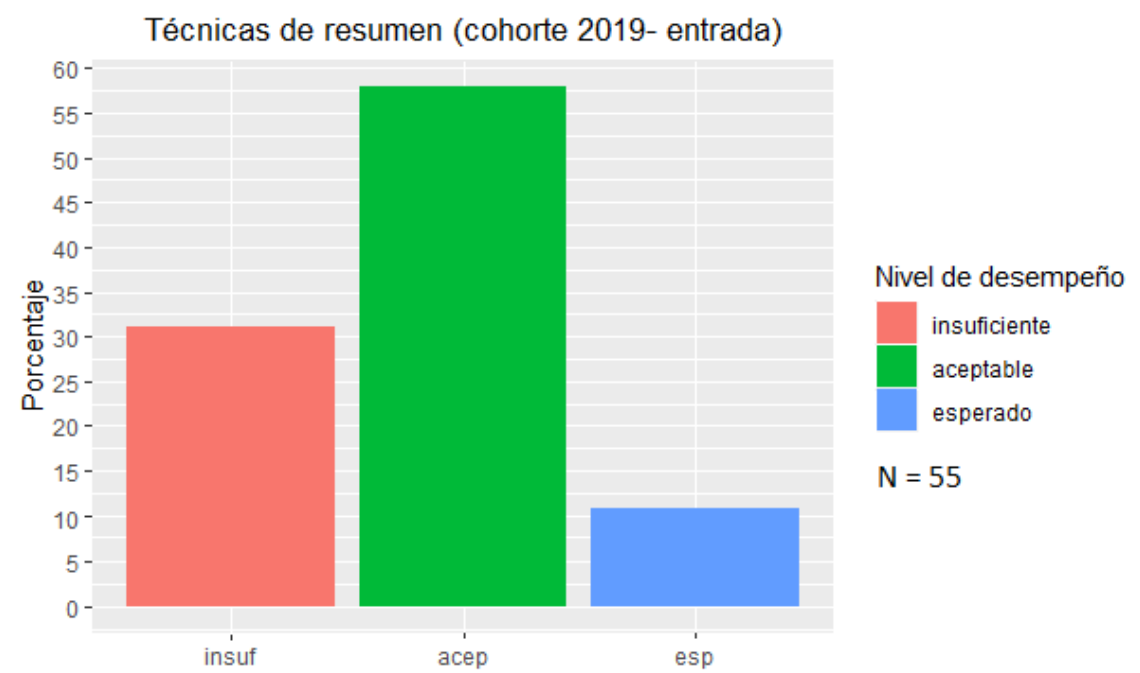

Gráfico 8 - Técnicas de resumen. Cohorte 2019 - Prueba de salida

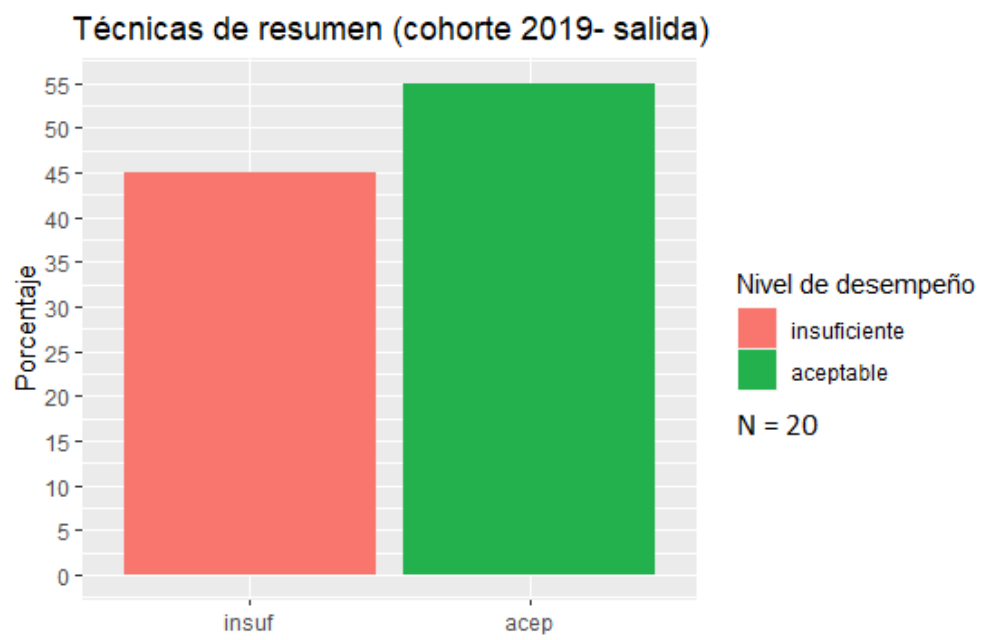

En la realización de los resúmenes pueden mencionarse diversas cuestiones. Hay comportamientos equiparables en algunas de las categorías evaluadas entre las pruebas de entrada y las de salida, como ser en los resultados globales del uso de macrorreglas que son similares en el nivel aceptable ( $71 \%$ en las de entrada y $75 \%$ en las de salida), y en la observación una a una este mismo nivel siempre está en $55 \%$ o más, salvo en seleccionar donde se observa mayor dificultad (gráficos 9 y 10). En calidad discursiva se observa un comportamiento semejante la utilización de marcadores del discurso en los tres niveles de esperado, aceptable e insuficiente entre las pruebas de entrada y las de salida. 
Gráfico 9 - Resumen de texto. Cohorte 2019 - Prueba de entrada

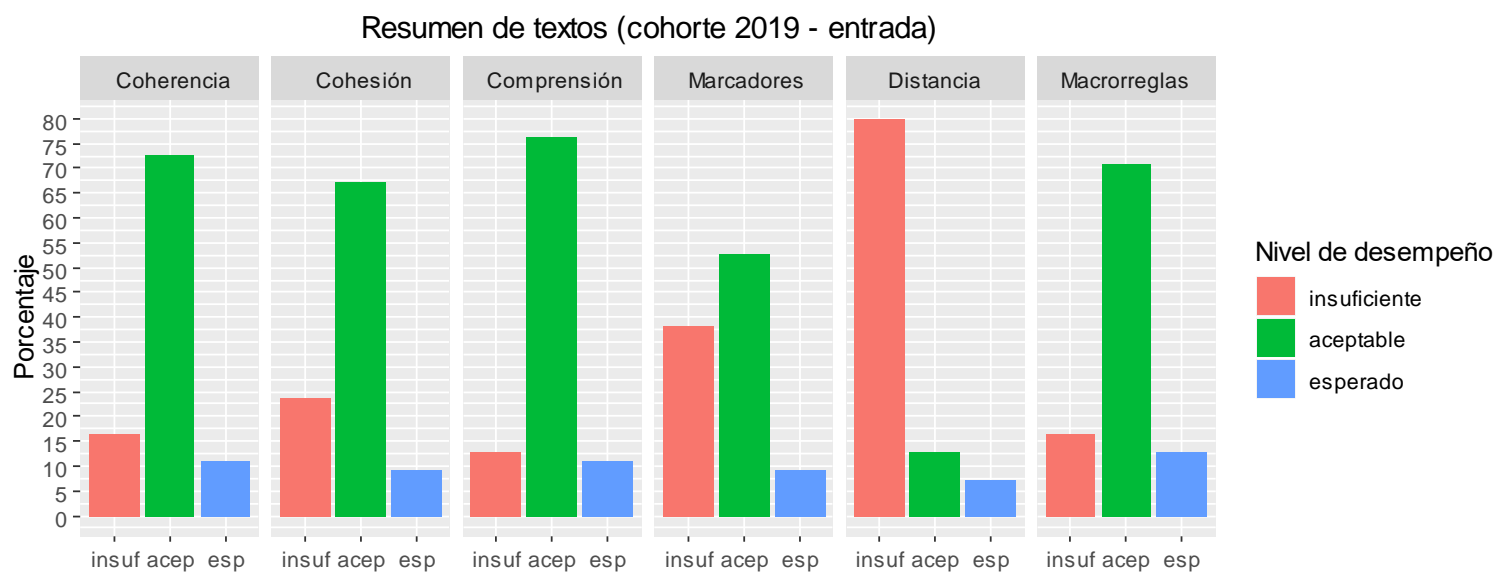

Gráfico 10 - Resumen de texto. Cohorte 2019 - Prueba de salida

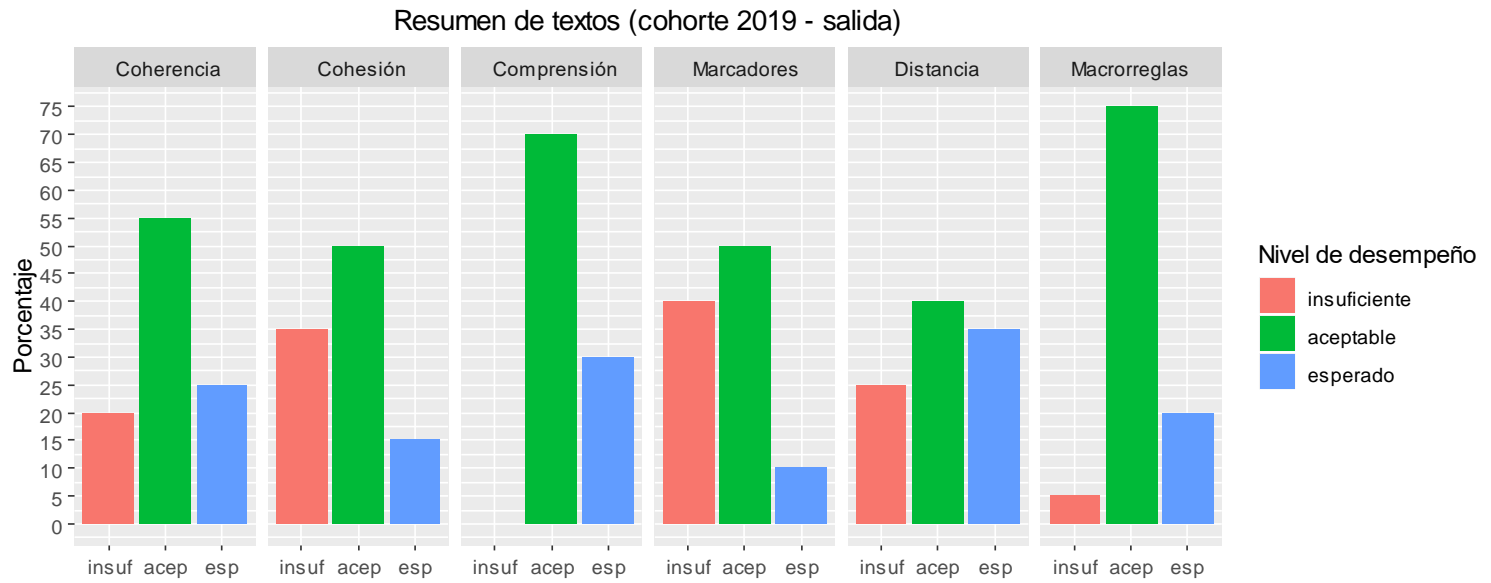

En cuanto al reconocimiento de cambios significativos, se observó en distancia del texto una clara mejoría al bajar el nivel de insuficientes del $80 \%$ en la prueba de entrada al $25 \%$ en la de salida, diferencia que se repartió entre los resultados aceptables y los esperados. Así mismo, en calidad discursiva la categoría comprensión denota cambios a destacar ya que no aparece representado el nivel de insuficiente, y mejora el de esperado del $11 \%$ al $30 \%$.

\section{Resultados en resumen: comparación entre cohortes}

En las pruebas de entrada de las cohortes 2018 y 2019 es evidente que en ambas los resultados reflejan un uso aceptable de las macrorreglas, predominando los resultados aceptables (58\% y $70 \%$ respectivamente). La mayor diferencia de comportamiento entre ambas cohortes se da en las pruebas de salida en las que se observó que los resultados son 
distintos y con una evolución inversa ya que mejoran notablemente para la cohorte 2018, mientras empeoran en la 2019. En la primera cohorte desaparecen los insuficientes, disminuyen los aceptables y predominan los esperados (62\%), mientras que en la segunda se mantiene el porcentaje de aceptables, aumentan los insuficientes (45\%) y desaparecen los esperados. Es decir que se produce una clara mejoría en la cohorte cuyos estudiantes tuvieron un semestre más entre ambas pruebas y el acompañamiento de la cátedra de ILEA.

En cuanto a la calidad discursiva entre ambas cohortes se observa un comportamiento diferente, siendo más favorables los resultados de la cohorte 2018, los cuales a su vez eran los más destacados respecto al resto de las cuestiones evaluadas, y mejoraban significativamente en la prueba de salida con un $75 \%$ de resultados esperados en tres de las cuatro categorías. En cambio para la cohorte 2019 los resultados no son tan favorables, así como tampoco es tan notoria la mejoría entre la prueba de entrada y la de salida, manteniendo en ambas porcentajes de resultados insuficientes que en cohesión llega al $30 \%$ y en marcadores del discurso al $40 \%$. Finalmente, en distancia del texto, en ambas cohortes se observa una mejoría reflejada en la disminución del porcentaje de resultados insuficientes, ya que para la cohorte 2918 baja del 72\% al 12\% entre la prueba de entrada y la de salida, mientras que en el 2019 baja del $80 \%$ al $25 \%$.

La comparación entre cohortes en el uso de macrorreglas y en calidad discursiva demuestra que hay diferencias netas que dan cuenta de un proceso de mejoramiento y consolidación de los aprendizajes, entre las pruebas de entrada y de salida; sin embargo es claramente más significativo el mejoramiento para la cohorte 2018. En el análisis de los posibles factores que incidieron se consideró no solo la cuestión del transcurso de un período más de estudio -el segundo cuatrimestre de primer año-, sino la colaboración de la cátedra de ILEA acompañando diferentes actos de escritura, en particular el desarrollo durante un bimestre del proceso de preparación del Encuentro de estudiantes y docentestutores, simulacro de congreso con que, desde el 2016, se cierra el ciclo lectivo de primer año (DIEZ et al., 2017). El mismo incluye todas las instancias tanto de organización, como de escritura de un resumen a partir de un informe técnico-, revisión por parte de un comité científico y presentación de un poster académico -incluye un bosquejo preliminar y pautas de diseño-.

\section{Discusión}

Entre los resultados de esta investigación se evidencia que, para la cohorte 2018, en la prueba de entrada los estudiantes manifestaron mayores dificultades al expresar las técnicas que habían utilizado para resumir, que en construir el texto, obteniendo más de la mitad la valoración de insuficiente. Además, se observó poco nivel de profundidad en las respuestas y una disminución de estas respecto a la escritura del resumen. Esto sucedió aun 
cuando no se les solicitó una explicación teórica de las macrorreglas a la que ningún estudiante hizo referencia, sino que expresaran a partir de su propia práctica cómo lo habían realizado. Lo anterior puede ser una consecuencia del escaso reconocimiento y reflexión acerca de la construcción de la propia escritura (HENAO, et al., 2011).

No obstante, en las pruebas de salida mejoraron el rendimiento al explicar la técnica de resumir, predominando resultados aceptables y desapareciendo los insuficientes, lo cual permitiría señalar que es probable que los estudiantes no contaron, en su proceso de formación inicial, con un acercamiento a la elaboración del resumen desde una perspectiva sociocognitiva (DIDACTEXT, 2003), posiblemente, resaltando aseveraciones desde una perspectiva tradicional donde se entiende el resumen como contar con sus palabras lo que entendió en el texto como lo describen Castañeda y Henao (1995), desaprovechando el potencial metacognitivo y metalingüístico que lleva consigo la elaboración del resumen a través de las macrorreglas (LONDOÑO, 2016).

Este apego al texto que se busca resumir y que lleva a la copia o plagio, demuestra la dificultad en la construcción de paráfrasis que permiten las reformulaciones sintácticas del texto -va más allá de la mera reformulación léxica- e implica bajos niveles de comprensión lectora. Resulta complejo abordar las construcciones textuales con plagio por la diversidad de niveles que pueden tener -inclusive inconscientes-, las que suelen estar consolidadas no solo en los y las alumnas, sino también entre los y las docentes (ROIG, 1999 y 2001).

En el desempeño de esta misma cohorte los resultados globales para la actividad de resumir un artículo de divulgación, también evidencian un mejoramiento entre las pruebas de entrada y las de salida que resultó significativo, haciendo un mejor uso de las macrorreglas, pese a no reconocerlas como tales en la explicación de cómo realizar un resumen. Por un lado, aumentaron los resultados esperados que constituye el nivel de desempeño más alto, siendo que en 10 de 11 aspectos evaluados superaron el 60\%, mientras que desaparecieron los resultados insuficientes en casi todas las categorías analizadas. La variable que registra un comportamiento diferente es la referida a toma de distancia con el texto al elaborar el resumen, la cual aún registra un índice reducido de insuficientes; sin embargo, un alto porcentaje de resultados son valorados como esperados. Esta toma de distancia potencia los procesos relacionados con la intertextualidad (HODGES, 2015), facilitando la apropiación la construcción argumentativa a través del manejo de las voces de los autores (RUIZ, et al., 2015), la elaboración de citaciones y referencias (MILARD, 2010), y su papel discursivo (GARCÍA, 2018).

Así mismo, la calidad discursiva se afianzó notablemente, evidenciándose que, más de la mitad obtuvo resultados aceptables en el uso de los marcadores discursivos; además, las valoraciones de coherencia, cohesión y comprensión de significados son positivas (MUÑOZ; OCAÑA, 2017). En cuando a esta mejoría significativa en la prueba de salida de la cohorte 2018, cabe destacar que la misma se realizó habiendo transcurrido un año desde el ingreso a la Universidad y con el acompañamiento de la cátedra ILEA también en el segundo 
cuatrimestre. En este sentido, los resultados de los textos son casi en su totalidad, aceptables y esperados, siendo también mejor el rendimiento en las técnicas de elaboración del resumen.

En la cohorte 2019, si bien los resultados en la prueba de entrada fueron mejores a los de la cohorte 2018 , en la prueba de salida no se evidencian cambios tan positivos. En la explicación de las técnicas utilizadas para resumir, más de la mitad obtuvo la valoración aceptable, tanto en la entrada como en la salida, siendo elevado el porcentaje de insuficientes. Al realizar el resumen también el porcentaje de resultados aceptables es alto ascendiendo a más del $70 \%$ en el uso de macrorreglas. Sin embargo, pese a estos resultados, el $80 \%$ no logra distanciarse del texto como también se evidenció en la otra cohorte, y si bien baja la mitad en la prueba de salida, el problema persiste.

Aun cuando en la mayoría de las variables evaluadas la valoración de aceptable está por encima de la mitad en ambas pruebas, el nivel de insuficientes en las de salida sigue manifestándose en varias categorías por encima del $20 \%$, siendo más elevado en el uso de la macrorregla integrar, en cohesión, en distancia del texto y en marcadores del discurso. Al respecto, podría ser posible que la fijación de los estudiantes por la copia tenga su origen en la impronta de las acciones de copiar durante la escolaridad, práctica que continúa estando extendida y que no demanda reflexión o crítica, diluyendo la presencia (o voz) del estudiante en su propia producción (AGRA DE BRITO NEVES, 2019).

Cabe aclarar que para esta segunda cohorte la prueba de salida se realizó habiendo transcurrido solo el primer cuatrimestre, a diferencia de lo ocurrido en la cohorte 2018. Por lo tanto, los y las estudiantes no tenían tanto trayecto universitario recorrido con el acompañamiento de ILEA para consolidar y ampliar los conocimientos y competencias en alfabetización académica durante la segunda mitad del ciclo académico. Por tanto, es factible afirmar que a medida que los estudiantes se apropiaban de los conceptos propios de la disciplina (HENAO, et al., 2010), sus niveles de comprensión y producción textual se afianzan (CARLINO, 2011), permitiéndoles acercarse con mayor confianza al texto y a la información allí consignada, empoderándose para la elaboración de diferentes géneros académicos o profesionales relacionados con su área disciplinar (HENAO, et al., 2017).

\section{Conclusiones}

En Argentina, de manera semejante a la situación en otras universidades de América Latina, se manifiestan problemas de alfabetización académica en estudiantes que ingresan al sistema educativo universitario; situación que también resulta un desafío en la Universidad Nacional de Río Negro. De ahí que esta problemática se viene analizando, permitiendo implementar diversas formas de intervención para abordarla. En las carreras de Geología y de Paleontología resulta una preocupación sostenida, lo que ha permitido fortalecer la 
perspectiva de la materia de Introducción a la Lectura y Escritura Académica como transversal a otras asignaturas de primer año y diseñándola desde lo que requieren para los demás campos de saber, e iniciando a los y las estudiantes en competencias de metacognición. Así mismo, pasó a concebirse como un Área de Comunicación Múltiple dentro de un Programa de Innovación Pedagógica en Geociencia, iniciado en 2016. A partir de estas decisiones expande sus acciones tanto docentes como pedagógicas, integrándose al desarrollo de experiencias educativas en las que se vuelven relevantes los procesos de lectura, escritura y oratoria, con la finalidad de elevar la calidad comunicativa. Entre estas experiencias se destaca la propuesta de un evento anual como cierre de cada ciclo lectivo, el "Encuentro de docentes y estudiantes de Geociencias", diseñado como simulacro de un congreso científico.

Entre los problemas a resolver para acortar el tiempo de mejoramiento de las capacidades comunicativas en los ingresantes se destaca el de reconocer los procesos que utilizan en los distintos tipos de lectura y escritura, así como el conocimiento y aplicación de marcos teóricos que colaboran en estas prácticas (e.g. macrorreglas de resumen); más allá de las correspondientes a la cultura académica y científica de las disciplinas, las que deberán incorporar con la orientación de los docentes de las mismas. Esto va unido al desarrollo de competencias autoevaluativas, metacognitivas y metalingüísticas, considerando que las mismas podrían constituir un factor de difícil discernimiento que está actuando como obstáculo cognitivo.

En cuanto a los problemas específicos que se detectaron al resumir, cabría centrarse en resolver la debilidad en tomar distancia del texto para construir el propio, debiendo los y las estudiantes apropiarse de las formas y estrategias para lograrlo, lo cual les permitiría superar la tendencia a ser textuales afectando tanto la calidad analítica de la lectura como el estilo de la propia escritura, permitiendo además superar caer en el plagio producto de no contar con estos dominios.

Los resultados altamente favorables en la prueba de salida de la cohorte 2018 permiten plantear una nueva idea frente a los factores que incidieron más allá de haber transcurrido un semestre más. En este sentido, se arribó a una nueva hipótesis acerca del nivel de incidencia que pudo tener en la alfabetización académica la integración de la cátedra ILEA en la segunda mitad del año para colaborar con los y las estudiantes, así como con los y las docentes de las materias curriculares. Esto estaría confirmando la importancia de la propuesta de innovación pedagógica iniciada en estas las carreras de Geología y de Paleontología, especialmente para primer año, centrada en la interdisciplina entre materias, así como en la construcción transdisciplinaria de objetos de aprendizaje, como factor relevante del mejoramiento de la comprensión lectora y la calidad de la producción textual. Así mismo, favoreciendo el reconocimiento de la relevancia de la alfabetización académica no solo de parte de los y las estudiantes, sino de los propios docentes, reconociendo en estos procesos de mejoramiento los problemas de la cultura escrita disciplinar y laboral, y las 
diversas maneras de resolverlos de una manera planificada durante los trayectos formativos iniciales.

\section{Referencias}

AGRA DE BRITOS NEVES, C. Les genres académicques au ProFis: un rapport d'experiénce et d'inclusión sociales à l'Unicamp. Linguagem \& Ensino, v. 22, n. 3, p. 733-751, jul- set. 2019. https://doi.org/10.15210/rle.v22i3.16678

ARGAÉZ, R.; GÓMEZ ESPINOSA, C. Tras la huella delatora. ¿Cómo ves? Revista de divulgación de la Ciencia de la UNAM, n. 71, octubre, p. 11-14, 2004.

ARGENTINA, GOBIERNO DE RÍO NEGRO, MINISTERIO DE EDUCACIÓN Y DERECHOS HUMANOS. Diseño curricular - Escuela Secundaria (Res. 945-17), 2017.

AROCENA, R.; SUTZ, J. La universidad latinoamericana del futuro: tendencias, escenarios, alternativas. México: Unión de Universidades de América Latina, 2001.

BENGOCHEA, N.; LEVÍN, F. El estado de la cuestión. En: NATALE, L. (ed.). En carrera: Escritura y lectura de textos académicos y profesionales. Buenos Aires: Los Polvorines, 2012. p. 79-96.

BERDICHEVSKY, M. Minería: el reto de una mayor equidad de género, Latino minería.com. Columna, n. 8, marzo. 2017.

BOADO, M.; CUSTORIO, L.; RAMÍREZ, R. La deserción estudiantil universitaria en la Udelar y en Uruguay entre 1997 y 2006. Montevideo: Universidad de la República, 2011.

CÁBANA, M. C.; BÁEZ, A; CAMPOS AVENDAÑO, L.; DIEZ, M. A.; TUNIK, M.; CASADíO, S. Una introducción a la cartografía en primer año de Geología: TIC aplicadas, transdisciplina y enseñanza como proceso. En: XX CONGRESO GEOLÓGICO ARGENTINO, Tucumán, Artículo breve, Buenos Aires: Asociación Geológica Argentina, 2017, p. 8-9.

CARLINO, P. ¿Qué nos dicen las investigaciones internacionales sobre la escritura en la Universidad? En: MARTÍNEZ, S. (ed.). Democratización de la universidad: investigaciones y experiencias sobre el acceso y la permanencia de los/as estudiantes. Neuquén: EDUCO Universidad Nacional del Comahue, 2011. p. 109-140.

CARLINO, P. Escribir, leer, y aprender en la universidad. Una introducción a la alfabetización académica. Buenos Aires: Fondo de Cultura Económica, 2005.

CASTAÑEDA, L. S.; HENAO, J. I. La lectura en la universidad. Medellín: Editorial Universidad de Antioquia, 1995.

CATINO, M.; JUARROS, F. Debates y dilemas sobre el ingreso a la Universidad pública en Argentina. Argumentos y reflexiones en perspectiva latinoamericana (55-70). En: MARTíNEZ, S. (ed.). Democratización de la universidad: investigaciones y experiencias sobre el acceso y la permanencia de los/as estudiantes. Neuquén: EDUCO - Universidad Nacional del Comahue, 2011. p. 55-70.

CONEAU. Informe de evaluación externa. Universidad Nacional de Río Negro. 82. Buenos Aires: CONEAU, 2018. 
CUBO DE SEVERINO, L.; CASTRO DE CASTILLO, E. Introducción. En: Los textos de la ciencia. Principales clases del discurso académico-científico. Córdoba: Comunicarte, 2005. p. 15-20.

DI CAUDO, M. V. Transformaciones universitarias y cupos en Ecuador: entre equidad, meritocracia y desarrollo. Nómadas, 44, p. 167-183, 2016. https://doi.org/10.30578/nomadas.n44a9

DIDACTEXT. Modelo sociocognitivo, pragmalingüístico y didáctico para la producción de textos escritos. Didáctica. Lengua y literatura, n. 15, p. 77-104, 2003.

DIEZ, M. A.; SERRA, S.; VERA, D.; CAMPATELLA, D.; DÍAZ, I. Los eventos científicos y académicos como espacios de aprendizaje y evaluación: Experiencias de enseñanza en Geociencias en la UNRN. En: XX CONGRESO GEOLÓGICO ARGENTINO, 2017, Tucumán, Artículo breve, Buenos Aires: Asociación Geológica Argentina, 2017. p. 15-19.

DIEZ, M.A.; CASADíO, S. Historia en tiempo presente de la enseñanza e investigación en Ciencias de la Tierra en la Universidad Nacional de Río Negro. Revista de la Facultad de Ciencias Exactas, Físicas y Naturales. Nueva Serie, El nacimiento de la geología en la enseñanza universitaria, Buenos Aires, 5, Suplemento 1, agosto 2018. p. 141-151.

FALCONE, L.; STRAMAZZI, M. La medición de la deserción real y la actividad universitaria. En: MARTÍNEZ, S. (ed.). Democratización de la universidad: investigaciones y experiencias sobre el acceso y la permanencia de los/as estudiantes. Neuquén: EDUCO - Universidad Nacional del Comahue, 2011, p. 235-248.

FAUSTO, C.; TOJAR, J. C.; RÍOS, J. M. La Educación como vía de empoderamiento laboral y social de la juventud universitaria en México. REVESCO: Revista de estudios cooperativos, 129, p. 84-101, 2018.

FERNÁNDEZ, N.; PÉREZ, C. La educación superior latinoamericana en el inicio del nuevo siglo. Situación, principales problemas y perspectivas futuras. Revista Española de Educación Comparada, 27, p. 123-148, 2016. https://doi.org/10.5944/reec.27.2016.15044

GALLEGOS, E. A cuatro años de la nacionalización de YPF. Oro Negro. EXm, v. 22, n. 59, p. 3437, 2015.

GARCIA, M. M. Argumentación y puntos de vista evidenciales: Acerca del condicional citativo en el discurso periodístico y en el discurso científico. Boletín de lingüística, v. 30, n. 49, p. 86109, 2018.

GLUZ, N.; ROSICA, M. ¿̇Ingreso condicionado o condicionantes en el ingreso? El acceso al sistema universitario en contextos de fragmentación escolar. En: MARTíNEZ, S. (ed). Democratización de la universidad: investigaciones y experiencias sobre el acceso y la permanencia de los/as estudiantes. Neuquén: EDUCO - Universidad Nacional del Comahue, 2011, p. 71-108.

GONZÁLEZ, P.; TUNIK, M.; CASAdÍO, S.; CÁBANA, M. C.; DIEZ, M. A. Las rocas en Roca: estrategia didáctica de enseñanza en Geociencias. En: XX CONGRESO GEOLÓGICO ARGENTINO, agosto, 2017, Tucumán, Artículo breve, Buenos Aires: Asociación Geológica Argentina, 2017. p. 30-35. 
HENAO, J. I.; LONDOÑO, D. A.; FRIAS, L. Y. Leer y argumentar en un curso de lenguaje para estudiantes de primer año de Derecho de la Institución Universitaria de Envigado (Colombia). Revista Virtual Universidad Católica del Norte, n. 50, p. 162-182, 2017.

HENAO, J. I., LONDOÑO, D. A., FRÍAS, L. Y.; CASTAÑEDA, L. S. Niveles de literacidad de los estudiantes de Psicología de la Institución Universitaria de Envigado. Zona Próxima, n. 15, p. 54-77, 2011.

HENAO, J. I.; LONDOÑO, D. A.; FRÍAS, L. Y.; MARÍN, G. S. Análisis del discurso en la formación de lectores críticos, autónomos y democráticos. Envigado: El libro total, 2010.

HERNÁNDEZ, H. H.; MARTUSCELLI, J.; MOCTEZUMA, D.; MUÑOZ, H.; NARRO, J. Los desafíos de las universidades de América Latina y el Caribe: ¿Qué somos y a dónde vamos? Perfiles educativos, v. 37, n. 147, p. 202-217, 2015.

HODGES, A. Intertextuality in discourse. In: TANNEN, D.; HAMILTON, H; SCHIFFRIN, D. The handbook of discourse analysis. London; Wiully Blackwell, 2015 . p. 42-60. https://doi.org/10.1002/9781118584194.ch2

LONDOÑO, D. A. Análisis sociolingüístico de literacidad en jóvenes de la Institución Universitaria de Envigado. Revista Lasallista de investigación, v. 13, n. 1, p. 49-64, 2016. https://doi.org/10.22507/rli.v13n1a4

LONDOÑO, D. A.; BERMUDEZ, H. L. Niveles de literacidad en jóvenes universitarios: entrevistas cualitativas y análisis sociolingüístico. Revista Latinoamericana de Ciencias Sociales, Niñez y Juventud, v. 16, n. 1, p. 315-330, 2016. https://doi.org/10.11600/1692715x.16119

MARTÍNEZ, S. ¿lgualdad de oportunidades? Un desafío de la Enseñanza Superior. Estudiantes y mediaciones para la enseñanza. En: MARTíNEZ, S. (ed.). Democratización de la universidad: investigaciones y experiencias sobre el acceso y la permanencia de los/as estudiantes. Neuquén: EDUCO - Universidad Nacional del Comahue, 2011. p. 19-54.

MESSINA, J.; SILVA, J. Desigualdad del ingreso en América Latina: comprendiendo el pasado para preparar el futuro. Panorama general. Lima: Grupo Banco Mundial, 2017.

MILARD, B. Las citaciones científicas: redes de referencias en universos de referencias. El ejemplo de los artículos de química. Redes. Revista hispana para el análisis de redes sociales, v. 19, n. 2, p. 64-93, 2010. https://doi.org/10.5565/rev/redes.400

MONTREAL, C. La mujer en la minería. Comunidad Portal Minero, Santiago de Chile: Portal Minero, 2018.

MUÑOZ, Á. E.; OCAÑA, M. Uso de estrategias metacognitivas para la comprensión textual. Cuadernos de Lingüística Hispánica, n. 29, p. 223-244, 2017. https://doi.org/10.19053/0121053X.n29.2017.5865

NATALE, L. (Ed.). En carrera: Escritura y lectura de textos académicos y profesionales. Buenos Aires: Los Polvorines, 2012.

NAVARRO, F.; ABRAMOVICH, A. La reseña académica. En: NATALE, L. (ed.). En carrera: Escritura y lectura de textos académicos y profesionales. Buenos Aires: Los Polvorines, 2012. p. 39-60. 
ROIG, M. When college students' attempts at paraphrasing become instances of potential plagiarism. Psychological Reports, n. 84, p. 973-982, 1999.

ROIG, M. Plagiarism and paraphrasing criteria of college and university professors. Ethics \& Behavior, v. 11, n. 3, p. 307-323, 2001. https://doi.org/10.1207/S15327019EB1103 8

RUIZ, F. J; TAMAYO, O. E; MARQUEZ, C. La argumentación en clase de ciencias, un modelo para su enseñanza. Educação e pesquisa, v. 41, n. 3, p. 629-645, 2015. https://doi.org/10.1590/S1517-9702201507129480

SMINK, V. Por qué se ha duplicado el número de jóvenes que van a la universidad en América Latina (y cuál es el lado negativo de este fenómeno). BBC News, 18 de mayo de 2017.

SORIA, K.; ZÚÑIGA, S. Aspectos determinantes del éxito académico de estudiantes universitarios. Formación universitaria, v. 7, n. 5, p. 41-50, 2014. https://doi.org/10.4067/S0718-50062014000500006

STAGNARO, D.; DA REPRESENTACAO, N. El proyecto de intervención. En: NATALE, L. (Ed.). En carrera: Escritura y lectura de textos académicos y profesionales. Buenos Aires: Los Polvorines, 2012. p. 157-169.

TAPASCO, O., RUÍZ, F.; OSORIO, D. Estudio del poder predictivo del puntaje de admisión sobre el desempeño académico universitario. Revista Latinoamericana de Estudios Educativos (Colombia), v. 12, n. 2, p. 148-165, 2016.

UNIVERSIDAD NACIONAL DE RÍO NEGRO. Memoria institucional 2018. Viedma: Universidad Nacional de Río Negro, 2018.

UNIVERSIDAD NACIONAL DE RÍO NEGRO. Proyecto Institucional. Viedma: Universidad Nacional de Río Negro, 2018.

WEBER, V. De andamios y puentes para el acceso y la permanencia en la Educación Superior. Propuestas pedagógicas y entornos virtuales. En MARTíNEZ, S. (ed). Democratización de la universidad: investigaciones y experiencias sobre el acceso y la permanencia de los/as estudiantes. Neuquén: EDUCO - Universidad Nacional del Comahue, 2011. p. 141-166.

YUNI, J., MELENDEZ, C; DÍAZ, A. Equidad y políticas universitarias: perspectivas desde Latinoamérica. REDU: Revista de Docencia Universitaria, v. 12, n. 2, p. 41-58, 2014. https://doi.org/10.4995/redu.2014.5639

ZUNINO, C.; MURACA, M. El ensayo académico. En NATALE, L. (Ed.). En carrera: Escritura y lectura de textos académicos y profesionales. Buenos Aires: Los Polvorines, 2012. p. 61-78.

Recebido em: 02/11/2019.

Aceito em: 16/02/2020. 